\title{
Quadratic Assignment Problem and its Relevance to the Real World: A Survey
}

\author{
Ravi Kumar Bhati \\ PG Scholar \\ Department of Computer Science and \\ Engineering, \\ Maulana Azad National Institute of Technology, \\ Bhopal-462051,India
}

\author{
Akhtar Rasool \\ Assistant Professor \\ Department of Computer Science and \\ Engineering, \\ Maulana Azad National Institute of Technology, \\ Bhopal-462051,India
}

\begin{abstract}
The Quadratic Assignment Problem (QAP) is the well known and significant combinatorial optimization problem. For several decades, it has been of keen interest for researchers and its improvement is still in progress. QAP is very important because it plays an important role in various complex real world problems. In this survey some of the prominent applications of QAP are illustrated which have been optimally applied to real world problems in diverse areas. Here inherent descriptions of various applications are summarized by highlighting the shortcomings of their applications related to QAP. This paper gives the future directions and strong conclusions on the survey of quadratic assignment problem and justify that the performance improvement of QAP is important.
\end{abstract}

\section{Keywords}

Combinatorial optimization problem, Linear Assignment Problem, Quadratic Assignment Problem

\section{INTRODUCTION}

Quadratic Assignment Problem (QAP) is a combinatorial optimization problem and considered as one of the hardest known problems in existence. Optimization problem which involves discrete decision variables is called combinatorial or discrete optimization problem. In general, QAP is the problem of assigning the set of facilities to the set of locations where flow/weight is present between every pair of facilities and distance is available between every pair of locations [1]. The objective is to assign a set of facilities to locations so as to minimize the total assignment cost with the constraints that every facility is assigned only one location and every location is assigned only one facility.

For several decades QAP has been attracting scientific community as a major research area and several research developments intended for QAP are ubiquitously available as a guideline for improvement. Importance of QAP is straightforward about the fact that numerous real-world applications can be modeled as QAP [2].

The quadratic assignment problem (QAP) is not only tough problem, but it is also considered as the hardest among the NP-Hard category so far. Varieties of methods exist for solving QAP like exact methods, heuristics, metaheuristics, and greedy algorithms [3]. Exact algorithms for QAP exist for finding the optimal solution and guarantee their optimality. For example Branch \& Bound [4], Dynamic programming [5], Cutting plane method [6], Branch \& cut [7] have been successfully applied to QAP but they are confined to specific problem sizes. As a result, these exact algorithms do not play an important role when problem size increases and for large problem instances they require a huge amount of computational time as well as resources. Therefore, exact methods can be applied to easy problems or small instances of difficult problems. Heuristic are general guidelines or "rules of thumb" for obtaining feasible/good solution but not necessarily optimal solution to large problem instances of QAP in a reasonable amount of time, but there is no guarantee of finding the optimal solution. Specific heuristics and metaheuristics are the two prominent classes of heuristics. Specific heuristics are intended to solve a specific problem and/or instance while metaheuristics are considered as general purpose algorithms that can be applied to solve almost any optimization problem [3]. Greedy or constructive algorithms start from scratch (empty solution) and construct a solution by assigning values to one decision variable at a time, until a complete solution is produced. Some of the commonly used methods for solving QAP are NEH heuristic[8], 2-Opt, 3-Opt, Greedy 2-Opt, Greedy 3-Opt[9], Ant Colony Optimization (ACO) [10], Artificial Neural Networks (NN) [11], Genetic Algorithms (GA) [12], Scatter Search (SS)[13], Simulated Annealing (SA) [14], Tabu Search (TS) [15], [16] and Greedy Randomized Adaptive Search Procedure (GRASP) [17], Iterated local search algorithm[18].

Some of the popular applications of QAP are hospital layout[19], backboard wiring problem[20], campus planning model[21], economic problems[22], decision framework[23], archaeology[24], statistical analysis[25], reaction chemistry[26], numerical analysis[27] and dartboard design[28].

Hospital lay-out problem [19] consists of allocating particular locations to the clinics with the intent of minimizing the total distance being travelled by patients. Backboard wiring problem [20] consists of assigning the components meticulous locations on a backboard in such a way that minimizes the total length of wiring used. Campus planning problem [21] consists of planning the sites of buildings on a campus. The objective in campus planning is to minimize the total weekly walking distance between the buildings. Heffley (1980) [22] applied QAP to economic problems. Francis and White (1974) [23] developed a decision framework for assigning a new facility (police posts, supermarkets, schools) in order to serve a given set of clients. Krarup and Pruzan (1978) [24] applied it to archaeology and Hubert (1987) [25] used in statistical analysis. Forsberg et al. (1994) [26] used it in the analysis of reaction chemistry and Brusco and Stahl (2000) [27] in numerical analysis. 
In this paper, QAP is illustrated in detail through Section 2. After that contribution of QAP in various real world applications are discussed in section 3. The main objective of this paper is to define "why QAP is important?" and why the performance improvement of QAP is important for real world?" Section 4 outlines the complexity in solving QAP and major issues. Section 5 describes the research trends in QAP. Section 6 briefly concludes the survey and gives the future research directions regarding QAP.

\section{QAP}

Real world problems are divided into two categories, namely decision problems and optimization problem as shown in fig. 1. Any problem for which the answer is either zero or one is called a decision problem. Any problem that involves the identification of an optimal (either minimum or maximum) value of a given cost function is known as an optimization problem or it can be defined as the problem of finding the best solution from all feasible solutions. Optimization problems are further classified into two important categories i.e. discrete/combinatorial optimization problems and continuous optimization problems. Combinatorial optimization problems involve discrete decision variables while continuous optimization problems involve continuous decision variables. Quadratic Assignment Problem (QAP) is a combinatorial optimization problem which indicates that it involves discrete decision variables and finite search space (set of feasible solutions). The objective function \& constraints of QAP may take any one of the form (linear, nonlinear, non-analytic and black box etc) [3].

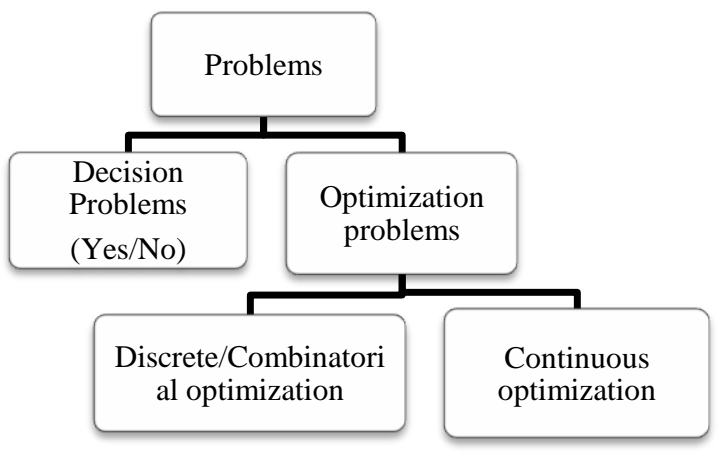

Fig 1: Problem formulation

QAP is a more complicated generalization of the Linear Assignment Problem (LAP). According to Hanan and Kurtzberg [29], LAP consists of assignment of $n$ people with $n$ jobs. Whenever an assignment is made, it includes the respective $\operatorname{cost} \mathrm{c}_{\mathrm{ij}}$, which indicates the assignment of a person $\mathrm{i}$ to job $\mathrm{j}$. The goal is to assign each person to one and only one job in such a way that minimizes the total cost, i.e., sum of each assignment cost.

Mathematically, LAP can be formulated as follows: we are given Cost matrix $(C)=\left[c_{i j}\right]$ of size $n \times n$. The goal is to minimize given objective function as follows:

$$
\min _{\varphi \in S_{n}}\left(\sum_{i=1}^{n} c_{i \varphi(i)}\right)
$$

where, $\mathbf{S}_{\mathbf{n}}$ is a set of all possible permutations of $(1,2, \ldots, \mathbf{n})$, $\varphi$ is one of the permutation belonging to $\mathbf{S}_{\mathbf{n}}$ and $\varphi(\mathrm{i})$ is the job assigned to person $\mathbf{i}$.
QAP was introduced by Koopmans and Beckmann in 1957 as a mathematical model for facility location problem. Their study was based on the assignment of locations to indivisible economic activities [30]. QAP is one of the basic computational problems in computer science and regarded as Non-Deterministically Polynomial (NP) Hard problem. It is the problem in which set of facilities is assigned to set of locations, with given distances between these locations and given flows/weights between the facilities. Our goal is to place these facilities on locations in such a way that total cost comes out to be minimized. Here total cost is referred as the sum of the products between flows and distances.

Mathematical formulation [2] of the QAP problem is as follows: we are given three $\mathrm{n} \times \mathrm{n}$ matrices namely Flow matrix $(A)=\left[a_{\mathrm{ij}}\right]$, Distance matrix $(\mathrm{B})=\left[\mathrm{b}_{\mathrm{ij}}\right]$ and Cost matrix (C) $=\left[c_{i j}\right]$, where usually $a_{i j}, b_{i j}>0$. The goal is to minimize given objective function as follows:

$$
\min _{\varphi \in S_{n}}\left(\sum_{i=1}^{n} \sum_{k=1}^{n} a_{i k} b_{\varphi(i) \varphi(k)}+\sum_{i=1}^{n} c_{i \varphi(i)}\right)
$$

where, $\mathbf{S}_{\mathbf{n}}$ is set of all possible permutations of $(1,2, \ldots ., n)$ and $\varphi$ is one of the permutation belonging to $\mathbf{S}_{\mathbf{n}}$ for which feasible solution turns out to be optimal. $\varphi(\mathrm{i})$ is the location assigned to facility $\mathbf{i}$ and $\varphi(\mathrm{k})$ is the location assigned to facility $\mathbf{k}$.

Here,

- $\mathbf{a}_{\mathbf{i k}}$ represents the flow from the facility $\mathbf{i}$ to the facility $\mathbf{k}$,

- $\mathbf{b}_{\mathbf{i k}}$ represents the distance from the location $\mathbf{i}$ to the location $\mathbf{k}$,

- $\quad \mathbf{c}_{\mathrm{ij}}$ represents the cost of the placing facility $\mathbf{i}$ to the location $\mathbf{j}$.

\subsection{Example of Quadratic Assignment Problem}

A simple example [31] of quadratic assignment problem is as follows:-

Here, four facilities which are to be assigned four locations. Facilities and locations are represented by 1,2,3,4 and A, B, C, D.

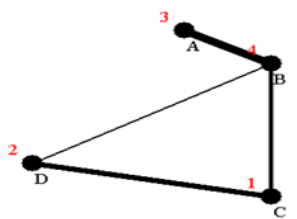

Flows

$\operatorname{Flow}(1,2)=3$

Flow $(1,4)=2$

$\operatorname{Flow}(2,4)=1$

Flow $(3,4)=4$

Optimal solution :-( $3,4,1,2) \rightarrow(A, B, C, D)$

Total cost $=395$ 


\section{APPLICATION AREAS}

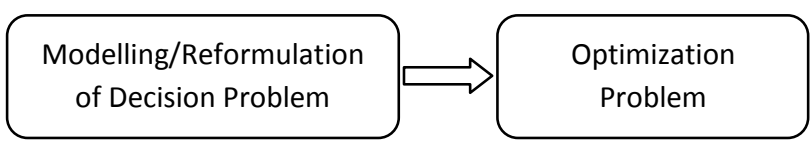

Fig 2: Problem formulation

Various applications pertaining to real world have been availing quadratic assignment problem as an optimal solution strategy is described here.

\subsection{Hospital layout problem}

In hospital layout problem, departments are assigned locations in order to minimize the total distance per year that is being travelled by patients. Formulation of the above problem using Quadratic Assignment Problem is presented [19] where various practical aspects of the problem are being discussed. A heuristic procedure for hospital layout [32] applied and consequently obtained optimal results. Planning of the hospital includes consideration of some vital factors like human and economic factors since the ease of patients should be primly considered.

Hospital Layout Problem comprises of assigning $\mathrm{n}$ locations (department) to $\mathrm{n}$ facilities (clinic) in a way of minimizing total distance travelled by patients. Here every pair of facilities has yearly flow and every pair of locations have distance which is denoted by $\mathrm{f}_{\mathrm{ij}}$ and $\mathrm{d}_{\mathrm{kl}}$ respectively. Each location can house only one facility and each facility occupies only one location. Hospital Layout Problem formulation as Quadratic Assignment Problem [19] is as follows:-

$$
\begin{gathered}
\min \varphi=\sum_{i k} \sum_{j l} f_{i j} d_{k l} y_{i k} y_{j l} \\
\sum_{j \in I} y_{i j}=1 \forall i \epsilon I \\
\sum_{i \in I} y_{i j}=1 \forall j \epsilon J \\
y_{i j}\left\{\begin{array}{l}
1 \text { if facility is located at } j \\
=0
\end{array}\right.
\end{gathered}
$$

where, $\mathrm{I}$ is the set of all facilities to be located and $\mathrm{J}$ is the set of all locations.

\subsection{Backboard wiring problem}

Backboard wiring problem [20] comprises of the placement of electronic components on computer backboard with a view to minimize total length of wiring used for interconnection of components. The minimized wiring will result in better computing time and cost effectiveness.

Anstreicher had later extended the backboard wiring problem to the Steinberg wiring problem [33] which comprised of the placement of 34 components on 36 different positions onto a computer backboard with a total of 2625 interconnections. Two dummy components are added so that the total number of positions on the backboard equals the total number of components.

Let $\mathrm{a}_{\mathrm{ik}}$ be the number of wires connecting component $\mathrm{i}$ to component $\mathrm{k}$, and $\mathrm{b}_{\mathrm{jl}}$ be the distance from component $\mathrm{j}$ to component 1 on the backboard, then the general Steinberg Wiring Problem (SWP) can be formulated as follows[34]:

$$
\min \sum_{i, j, k, l} a_{i k} b_{j l} x_{i j} x_{k l}
$$

s.t.

$$
\begin{aligned}
& \sum_{j} x_{i j}=1 \quad i=1,2 \ldots \ldots . n ; \\
& \sum_{i} x_{i j}=1 \quad j=1,2 \ldots \ldots n ; \\
& x_{i j} \in\{0,1\} \quad i, j=1,2, \ldots \ldots n ;
\end{aligned}
$$

where, $x i j=1$ if and only if component $i$ is placed at position $j$ on the backboard.

\subsection{Dartboard design}

Dartboard [28] is a game played in which best possible arrangement of numbers $1,2 \ldots 20$ around the dartboard is to be found out. There are $20 ! \approx 10^{18}$ total distinct arrangements and $\frac{20 !}{2 * 20} \approx 10^{17}$ distinct cyclic groups available in dartboard given below.

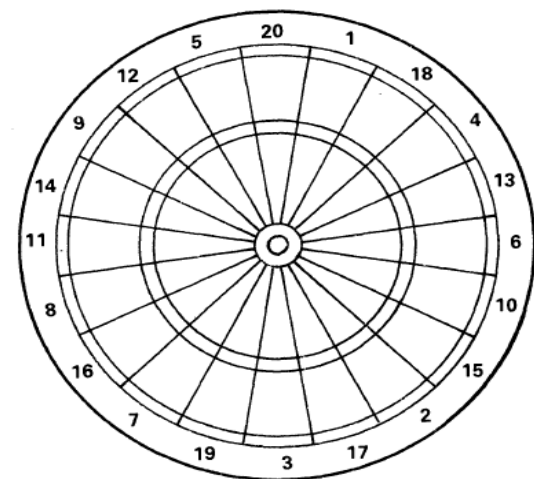

Fig 3: Dartboard design-the interior circle is called the bull's eye.

Under some assumptions, dartboard can be formulated as a quadratic assignment problem.

$$
\max z=\sum_{i=1}^{20} \sum_{j \neq 1} c_{i j} x_{i j}+\theta \sum_{i=1}^{20} \sum_{j \neq i} \sum_{\substack{l \neq i \\ l \neq j}} c_{i l} x_{i j} x_{j l},
$$

Here, 0 reflects the relative weight given to second-order deviations. Note that in the second term of the objective, the cost $c_{i l}$ is incurred only if for some $j$ the subsequence $(i, j$, appears in the permutation. This formulation is that of a maximum cost quadratic assignment problem (QAP). It should now be clear that allowing positive probabilities $P_{t}$ for $t>2$ would lead to an assignment problem of degree $t$ without sub-tours.

\section{COMPUTATIONAL COMPLEXITY}

S. Sahni and T. Gonzalez [30] already proved in 1976 that QAP is NP-Hard category problem. Consider an example of assigning $\mathrm{n}$ facilities to $\mathrm{n}$ locations such that each facility is assigned only single location and each location is assigned 
unique facility. To carry out this process, in general, there must be total possible permutations which are $n$ !. But when $n$ is large then $n$ ! turns out to be very large. For example [2], if 10 facilities are to be assigned to 10 locations, then total $\mathrm{n} !=3628800$ permutations to be checked which is not possible in practice as it requires a large amount of computational time.

$$
n !=n *(n-1) *(n-2) *(n-3) \ldots . .3 * 2 * 1
$$

Above expression leads to $\mathrm{O}\left(\mathrm{n}^{\mathrm{n}}\right)$ exponential time complexity which shows that solving instances of Quadratic Assignment Problem having large instances i.e. large value of $n$ subsequently turns out to be tedious in terms of time \& resource complexity. So heuristics and metaheuristics are used for finding a nearly optimal solution rather than exact solution for large instances of QAP.

\section{RESEARCH TRENDS}

This section highlights the available research trends with respect to time, for the period of 50 years after the QAP first appeared in the literature. Fig. 4 [35] shows the interest of researchers in QAP in the recent years. Till 2004 there is a steady increase after that in 2005 there is a moderate drop in the QAP research. The bibliography used in [35] lists 365 publications, of which about $95 \%$ deal directly with QAP. Fig. 5 encapsulates the various publications, which are classified by solutions methods/techniques namely exact methods, heuristics and metaheuristics.

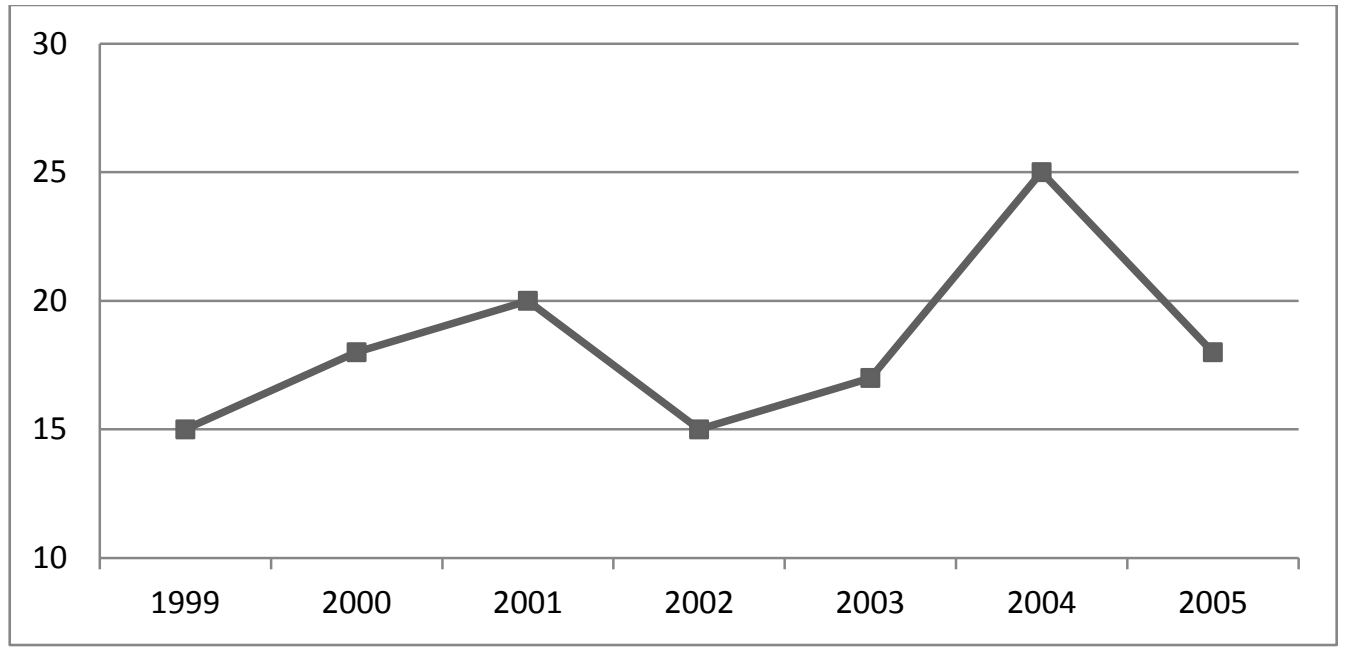

Fig 4: Number of QAP papers published in recent years

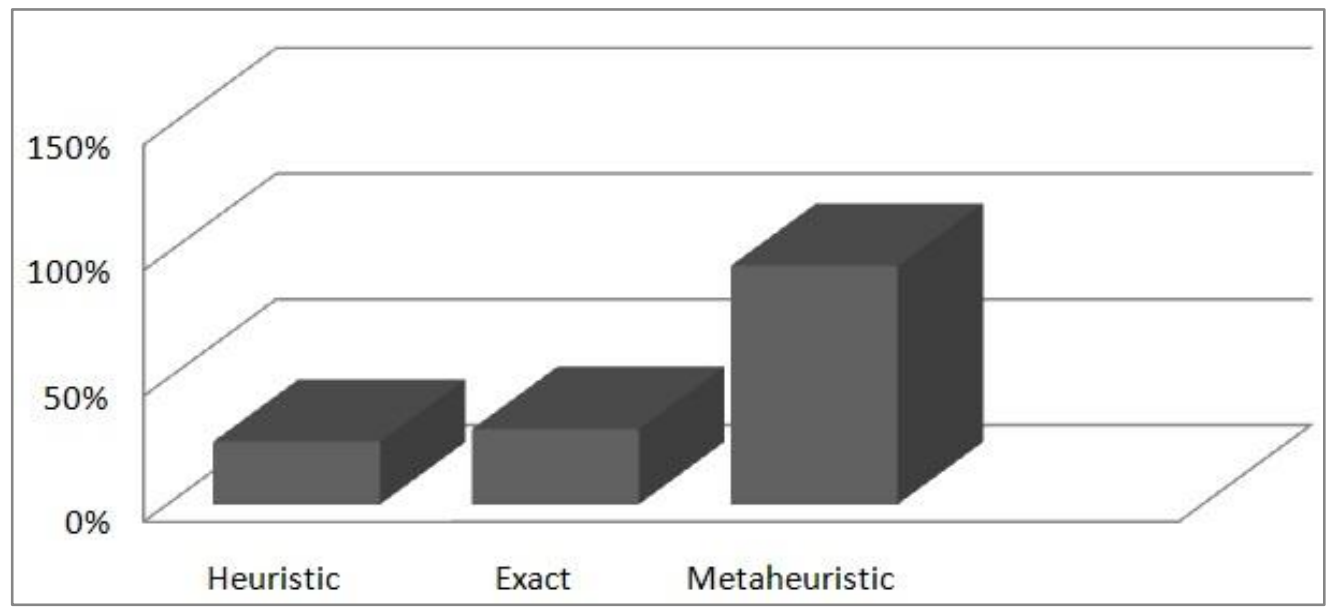

Fig 5: Solutions trends for QAP 


\section{CONCLUSION AND FUTURE SCOPE}

The Quadratic Assignment Problem (QAP) is a combinatorial optimization problem which has remained as a challenge for researchers when instance size grows. This survey paper highlights fundamentals of QAP with example and mathematical expression. Some of the prominent applications of QAP are discussed and later on complexity issues are discussed. The significance of QAP in a real world scenario is candid and straightforward from the applications described in this paper. Reasonably good insights have been shown regarding the applicability and importance of the QAP and this work can be directed towards inclusion of other real world applications which have been successfully modeled as QAP.

\section{REFERENCES}

[1] Burkard RE, Cela E, Pardalos PM, Pitsoulis LS, "The quadratic assignment problem: Handbook of combinatorial optimization", vol. 3. Kluwer; pp. 241337, 1998.

[2] Clayton W. Commander, "A Survey of the Quadratic Assignment Problem, with Applications", Morehead Electronic Journal of Applicable Mathematics Issue 4MATH-2005-01

[3] Talbi, El-Ghazali, "Metaheuristics: From Design to Implementation", Hoboken, N.J.: John Wiley \& Sons, 2009.

[4] Bazaraa, M.S., Kirca, O, "A branch-and-bound based heuristic for solving the quadratic assignment problem", Naval Research Logistics Quarterly 30, 287-304,1983.

[5] Christofides N., Benavent E., " An exact algorithm for the quadratic assignment problem", Operations Research 37 (5), 760-768,1989.

[6] Bazaraa, M.S., Sherali, H.D., "On the use of exact and heuristic cutting plane methods for the quadratic assignment problem", Journal of the Operational Research Society 33, 991-1003,1982.

[7] Padberg, M.W., Rinaldi, G., "A branch-and-cut algorithm for the resolution of large-scale symmetric travelling salesman problems", SIAM Review 33, 60100,1991 .

[8] Nawaz, M., Enscore Jr., E.E., Ham, I., "A heuristic algorithm for the m-machine, $n$-job flow-shop sequencing problem", OMEGA The International Journal of Management Science 11 (1), 91-95, 1983.

[9] Mahdi Bashiri and Hossein Karimi, "Effective heuristics and meta-heuristics for the quadratic assignment problem with tuned parameters and analytical comparisons", Journal of Industrial Engineering International 2012, 8:6.

[10] Vittorio Maniezzo and Alberto Colorni, "The Ant System Applied to the Quadratic Assignment Problem", IEEE transactions on knowledge and data engineering, VOL. 11, NO. 5, pp. 769-778, SEPTEMBER/OCTOBER 1999.

[11] Uwate, Y., Nishio, Y., Ushida, A., "Markov chain modeling of intermittency chaos and its application to Hopfield NN", IEICE Transactions on Fundamentals of Electronics Communications and Computer Sciences E87A (4), 774-779, 2004.
[12] Zvi Drezner, "A New Genetic Algorithm for the Quadratic Assignment Problem", INFORMS Journal on Computing, Vol. 15, No. 3, pp. 320-330, Summer 2003.

[13] Cung, V.-D., Mautor, T., Michelon, P., Tavares, A., “A scatter search based approach for the quadratic assignment problem", In: Proceedings of IEEE International Conference on Evolutionary Computation, pp. 165-169, 1997.

[14] Wilhelm, M.R., Ward, T.L., "Solving quadratic assignment problems by simulated annealing", IEEE Transactions 19, 107-119,1987.

[15] Glover, F., 1989a. Tabu search-Part I. ORSA Journal on Computing 1, 190-206.

[16] Glover, F., 1989b. Tabu search-Part II. ORSA Journal on Computing 2, 4-32.

[17] Oliveira, C.A.S., Pardalos, M.P., Resende, M.G.G., "GRASP with path relinking for the quadratic assignment problem In: Experimental and Efficient Algorithms", Third International Workshop (WEA 2004), Brazil, LNCS 3059. Springer, pp. 356-368, 2004.

[18] Ramkumar AS, Ponnambalam SG, Jawahar N, Suresh RK, "Iterated fast local search algorithm for quadratic assignment problems", Robot Comput Integr Manuf 2008. doi:10.1016/j.rcim.2007.01.004.

[19] A. N. Elshafei, "Hospital layout as a quadratic assignment problem", Operations Research Quarterly 28, 167-179, 1977.

[20] L. Steinberg, "The Backboard Wiring Problem: A Placement Algorith",. SIAM Review 3 37-50, 1961.

[21] Dickey, J.W., Hopkins, J.W., "Campus building arrangement using Topaz”, Transportation Research 6 , 59-68, 1972.

[22] Heffley, D.R., "Decomposition of the KoopmansBeckmann problem", Regional Science and Urban Economics 10 (4), 571-580, 1980.

[23] Francis, R.L., White, J.A., "Facility Layout and Location: An Analytical Approach", Prentice-Hall, Englewood Cliffs, NJ, 1974.

[24] Krarup, J., Pruzan, P.M., "Computer-aided layout design", Mathematical Programming Study 9, 75-94, 1978.

[25] Hubert, L., "Assignment methods in combinatorial data analysis", Statistics: Textbooks and Monographs Series, vol. 73. Marcel Dekker, 1987.

[26] Forsberg, J.H., Delaney, R.M., Zhao, Q., Harakas, G., Chandran, R., "Analyzing lanthanide-included shifts in the NMR spectra of lanthanide (III) complexes derived from 1,4,7,10-tetrakis ( $N, N$-diethylacetamido)-1,4,7,10tetraazacyclododecane", Inorganic Chemistry 34, 37053715,1994

[27] Brusco, M.J., Stahl, S., "Using quadratic assignment methods to generate initial permutations for leastsquares unidimensional scaling of symmetric proximity matrices", Journal of Classification 17 (2), 197-223, 2000.

[28] H. A. Eiselt and Gilbert Laporte, "A combinatorial optimization problem arising in dartboard design", The 
Journal of the Operational Research Society, Vol. 42, No. 2, pp. 113-118, Feb., 1991.

[29] Hanan, M. and J.M. Kurtzberg, "A Review of the Placement and Quadratic Assignment Problems", SIAM Review 14, 324-342, 1972.

[30] T. C. Koopmans and M. J. Beckmann, "Assignment problems and the location of economic activities", Econometrica 25, pp.53-76, 1957.

[31] http://www.neos-guide.org/content/qap4

[32] M. Motaghi, A. Hamzenejad, L. Riyahi, M. Soheili Kashani, "Optimization of Hospital Layout through the Application of Heuristic Techniques (Diamond Algorithm) in Shafa Hospital (2009)", Int. J. Manag. Bus. Res., 1 (3), 133-138, Summer 2011.
[33] Brixius, N.W. and K.M. Anstreicher, "The Steinberg Wiring Problem", To appear in, The Sharpest Cut, M. GrÄotschel, ed., SIAM, 2001.

[34] Clayton W. Commander, "A Survey of the Quadratic Assignment Problem, with Applications", Morehead Electronic Journal of Applicable Mathematics Issue 4 -MATH-2005-01.

[35] Eliane Maria Loiola, Nair Maria Maia de Abreu, Paulo Oswaldo Boaventura-Netto, Peter Hahn, Tania Querido, "A survey for the quadratic assignment problem", European Journal of Operational Research 176 (2007) $657-690$ 